
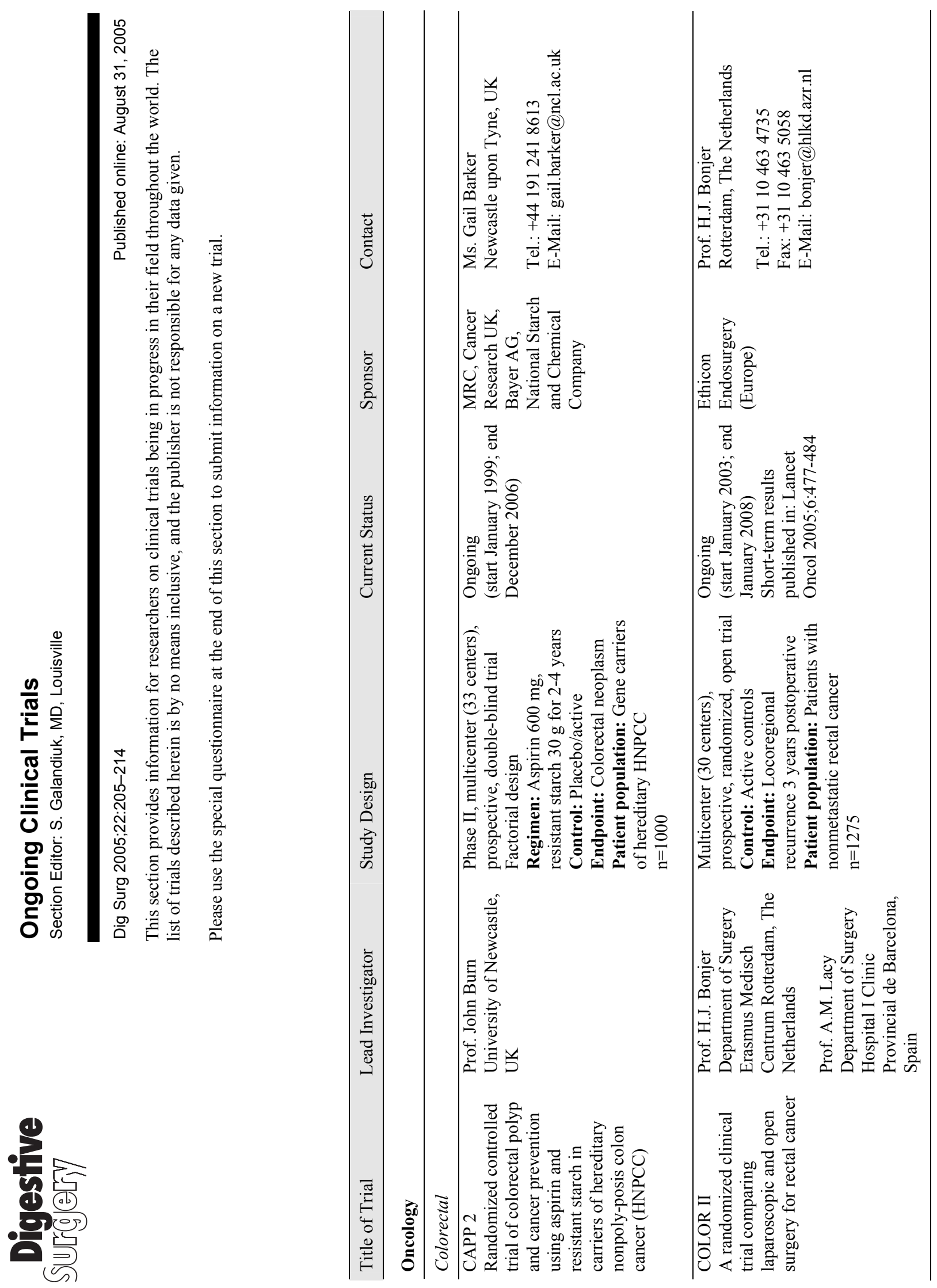


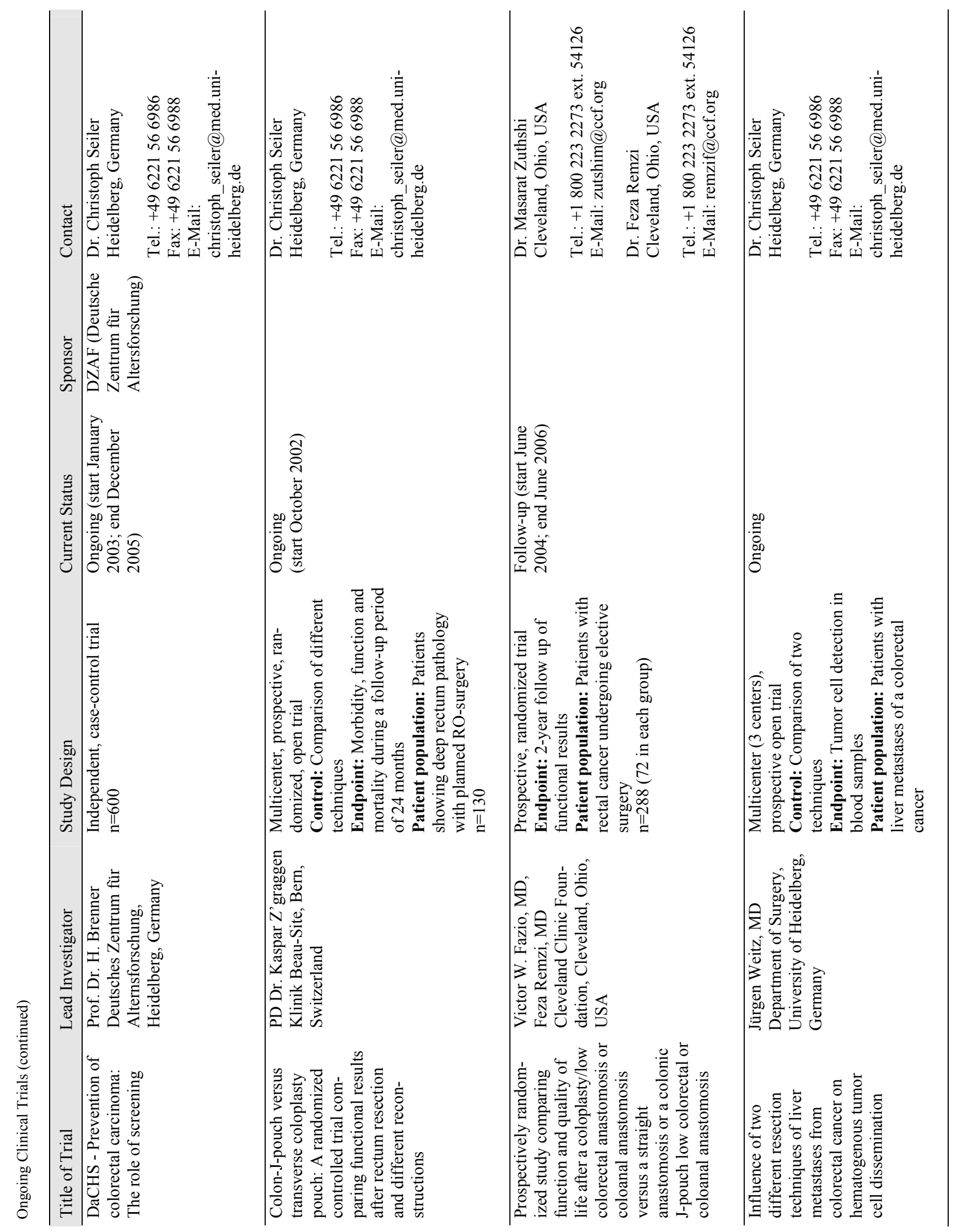




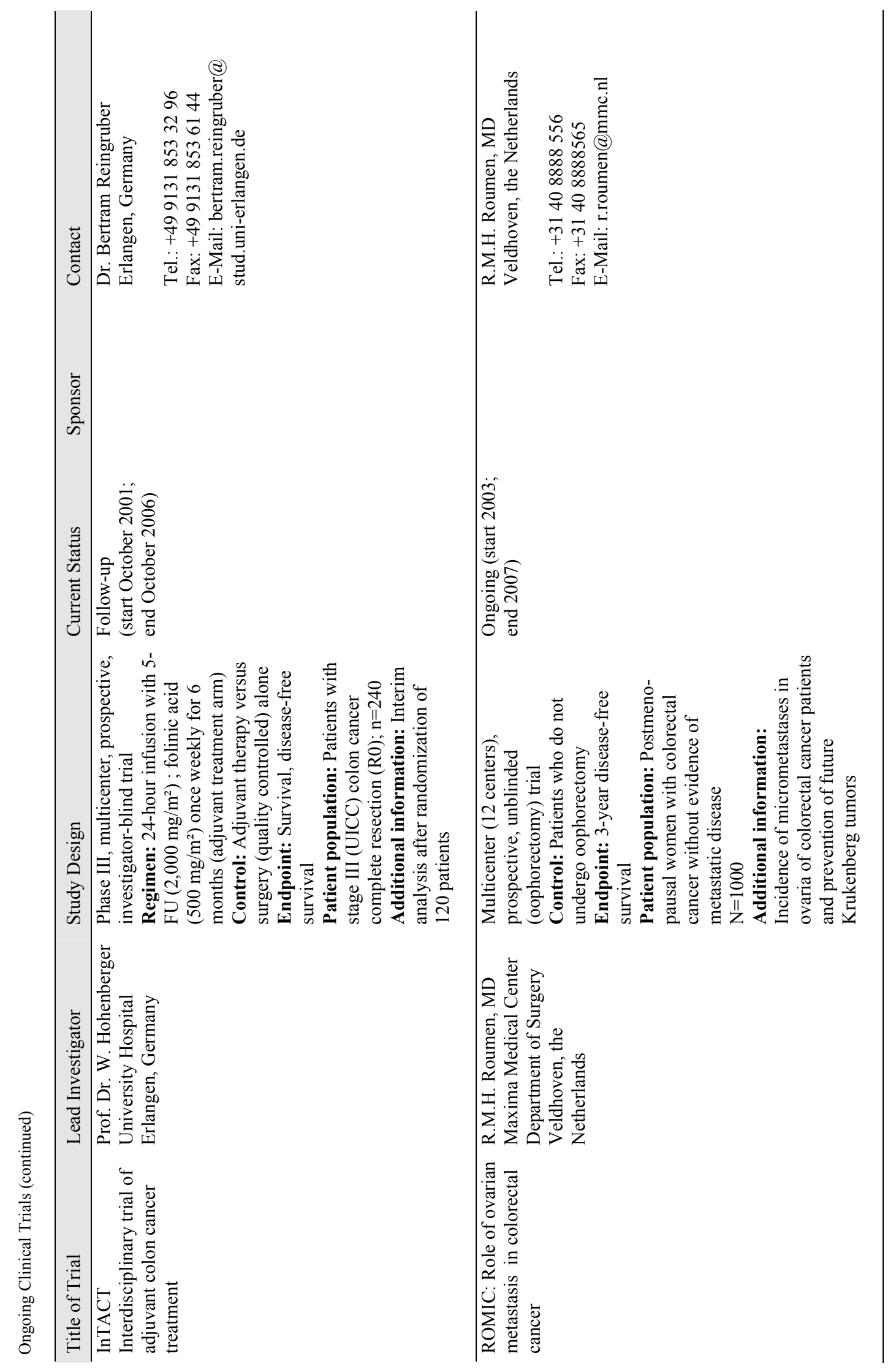




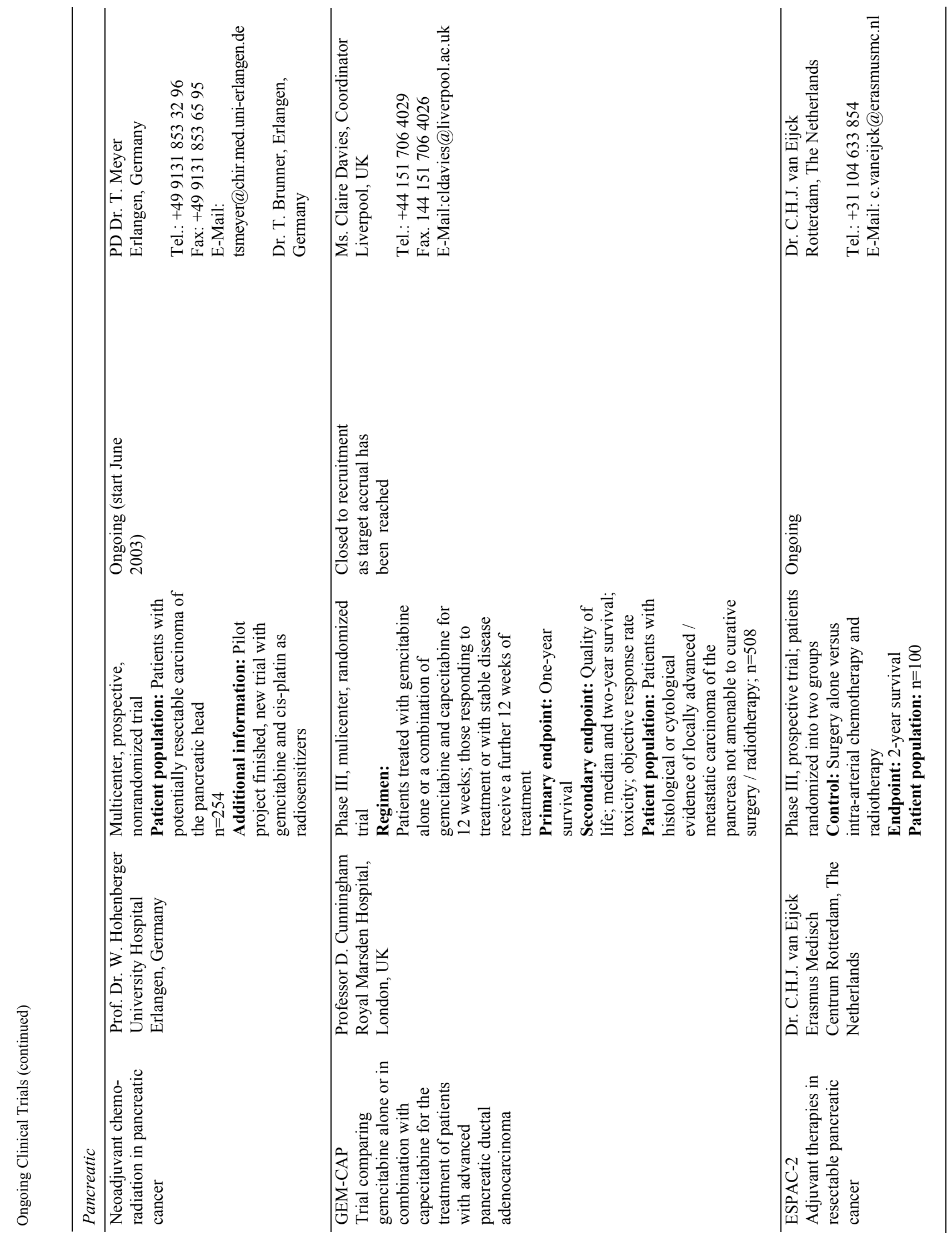



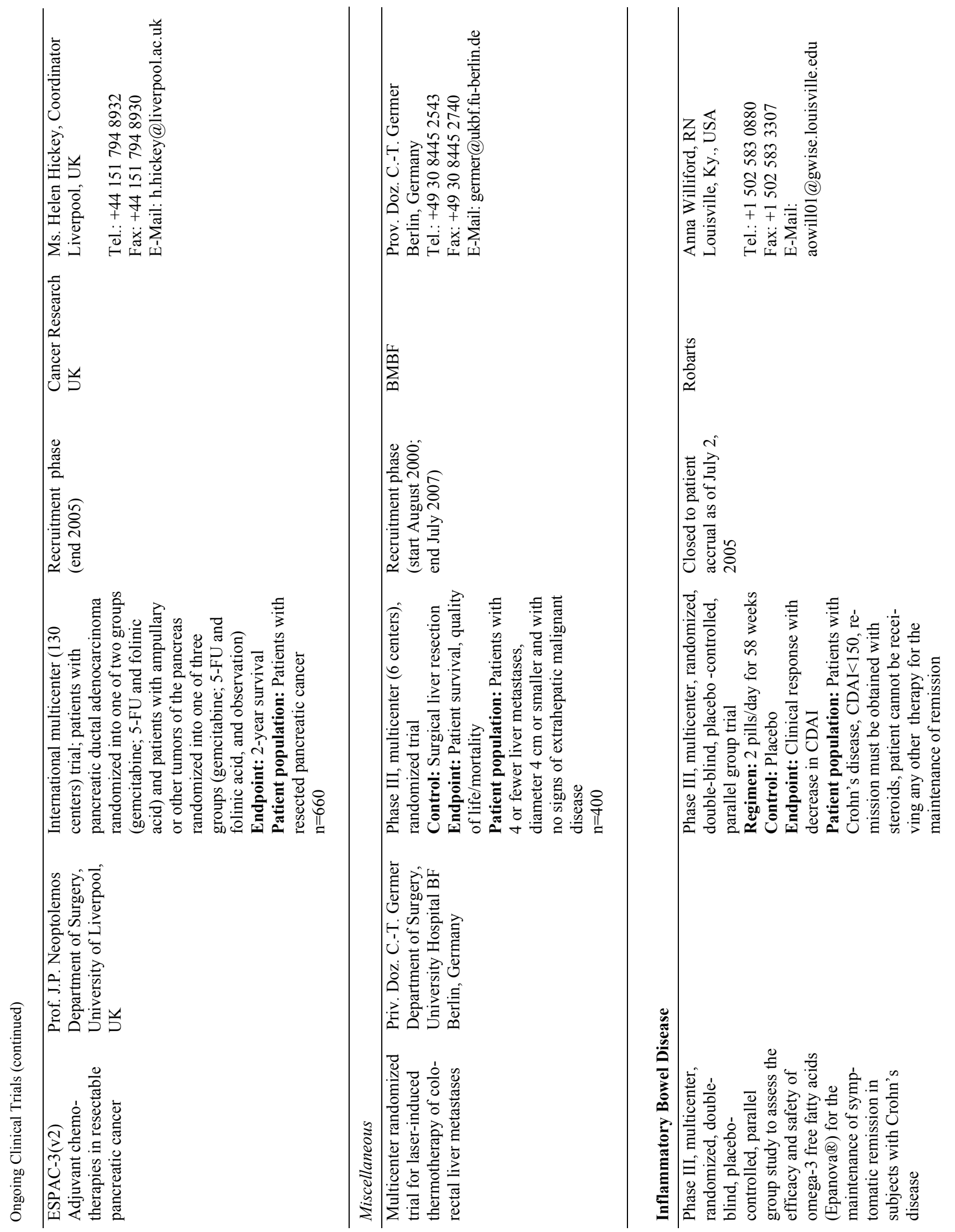


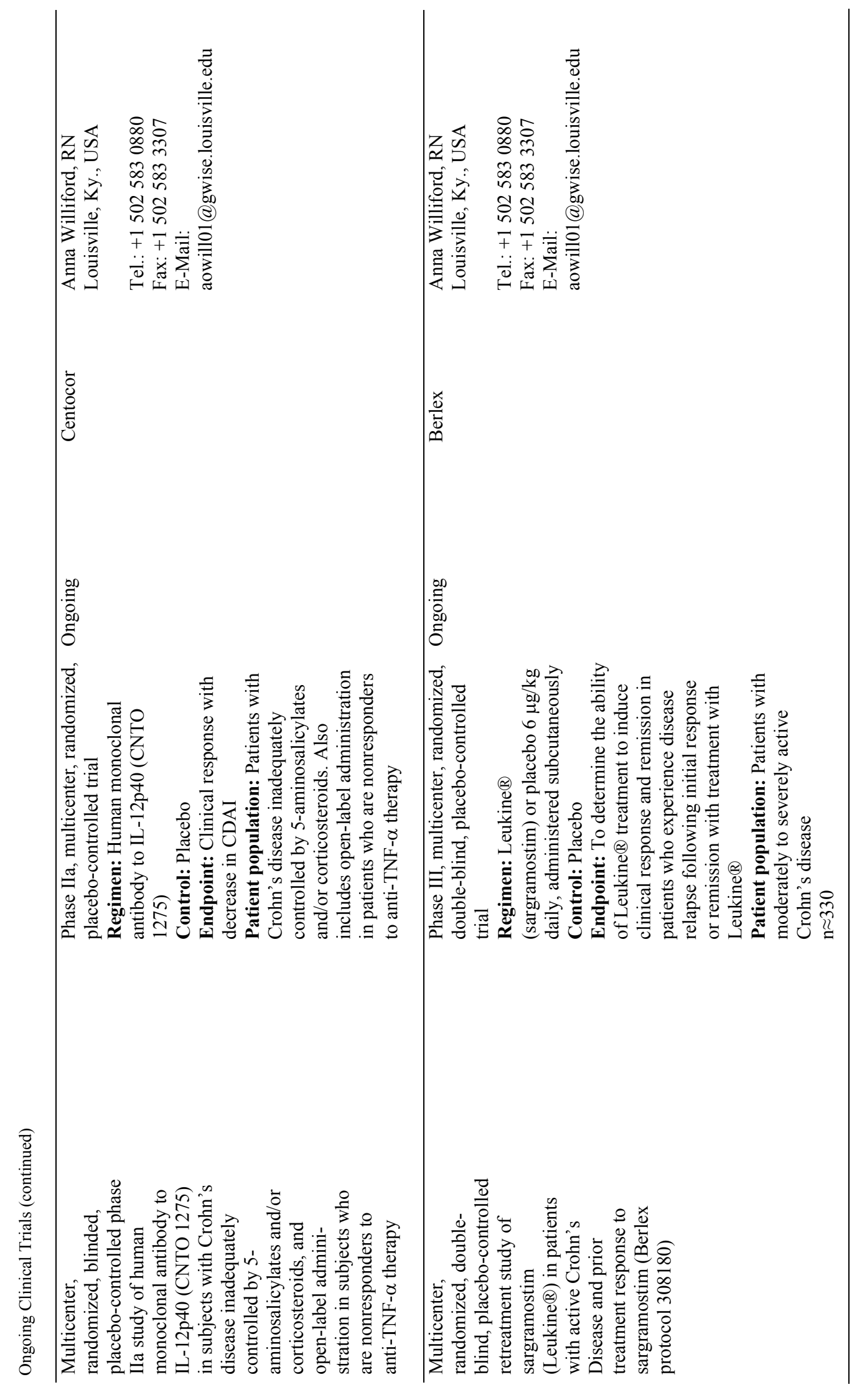




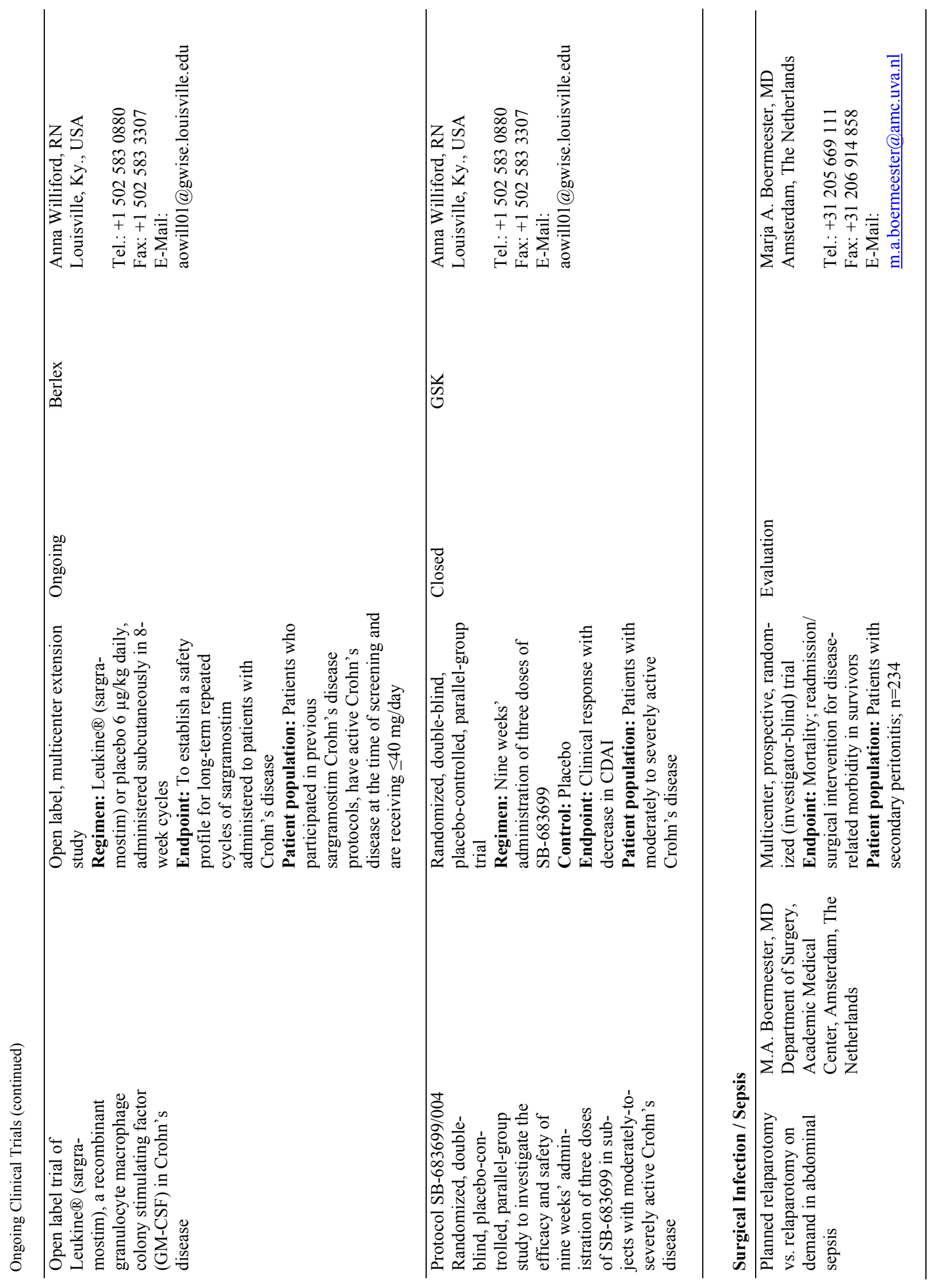



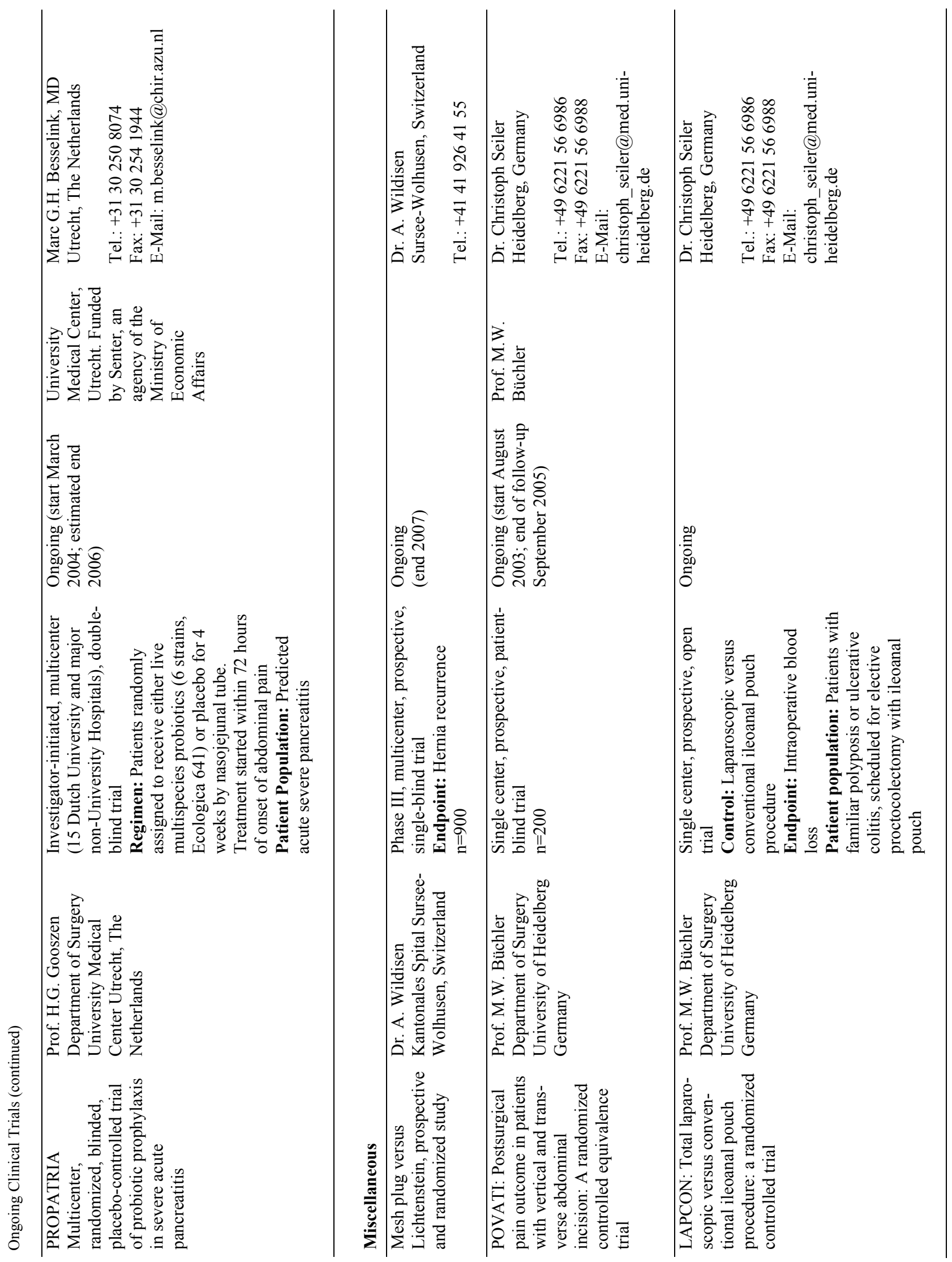


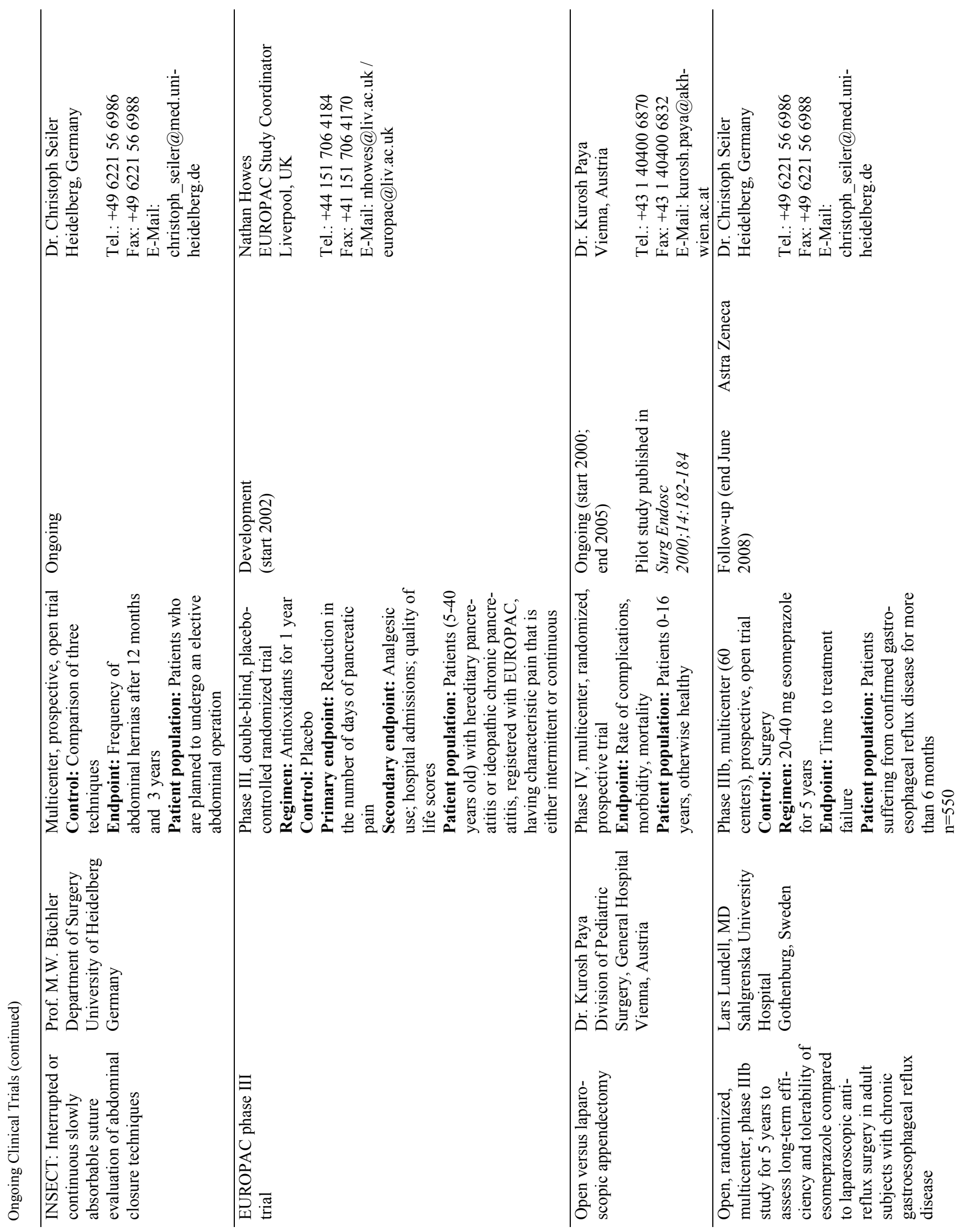


Digestive

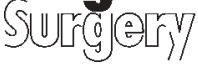

\section{Questionnaire \\ for Trial Submission}

To submit information on a clinical trial for publication in the 'Ongoing Clinical Trials' section, please complete this short questionnaire.

\section{1) Title of study (in full)}

\section{2) Lead investigator}

Title:

Name:

Affiliation:

\section{3) Field of study}

Please indicate one or more of the following categories:

$\square$ Oncology $\square$ Inflammatory Bowel Disease

$\square$ Motility $\square$ Surgical Infection/Sepsis $\quad \square$ Miscellaneous

$\underline{\text { Other (please specify): }}$

\section{4) Study phase (drug trials only)}

Please indicate one of the following categories:

$\square$ Phase I $\square$ Phase II $\square$ Phase III $\square$ Phase IV

$\square$ Independent (investigator initiated)

\section{5) Study design}

$\square$ Multicenter $\square$ Single center (please indicate as appropriate)

If multicenter, please state how many centers are involved:

$\square$ Prospective $\square$ Retrospective (please indicate as appropriate)

If prospective, level of randomization (e.g. single blind, double blind, investigator-blind):

Controls (i.e. placebo-, active-, etc.):

Cross-over design (one-way, two-way, etc.):

Duration of therapy (if applicable):

Dosing regimen (if applicable):

Primary study endpoint:

Other information on study design (please include any other details that might be relevant):

\section{6) Patients}

Number of patients (planned):

Patient population (e.g. patients with mild UC previously controlled on...):

\section{7) Current status of study}

Start date:

Estimated end date:

Status (please indicate as appropriate):

$\square$ Development $\quad \square$ Recruitment $\quad \square$ Ongoing

$\square$ LPO $\quad \square$ Analysis in progress

\section{Publication}

Abstract submitted to:

Manuscript submitted to:

8) Sponsor

9) Contact for further information:

Name:

Address:

Telephone:

Fax:

E-Mail:

Please return the completed questionnaire as soon as possible to the Section Editor:

Susan Galandiuk, MD

Department of Surgery

School of Medicine

University of Louisville

Louisville, KY 40292, USA

Tel.: + 15028525442

Fax: +1 5028528915 or contact:

S. Karger AG

Attn.: Ms. Yvonne Rebmann

Allschwilerstrasse 10

PO Box

CH-4009 Basel

Tel.: +41613061351

Fax: +41 613061234

E-Mail: y.rebmann@karger.ch 